EPJ Web of Conferences 110,01061 (2016)

DOI: $10.1051 /$ epjconf/201611001061

(C) Owned by the authors, published by EDP Sciences, 2016

\title{
THE INTERACTION POTENTIAL OF AN OPEN NANOTUBE AND ITS PERMEABILITY: MOLECULAR DYNAMICS SIMULATION
}

\author{
Mikhail A Bubenchikov ${ }^{1}$, Alexander I Potekaev ${ }^{1}$, Alexey M Bubenchikov ${ }^{1}$, Olesya V Usenko, a, \\ Alexander $V$ Malozemov ${ }^{1}$ and Egor A Tarasov ${ }^{1}$ \\ ${ }^{1}$ National Research Tomsk State University, 634050, 36 Lenin av., Tomsk, Russia
}

\begin{abstract}
The integration of the modified LJ-potential allowed revealing the universal effect of the open carbon tube on the molecular objects moving within or proximate to the tube. There has been established that there are modes of the molecule motion without the energy exchange with the atoms of the carbon framing, under which the moving molecules are subjected to the considerable activation in the tube. The potential holes being the sorption zones in fact are localized.
\end{abstract}

\section{Introduction}

Carbon nanotubes are used in various fields of human activity: in the pharmaceutical industry [1-3], microelectronics [4, 5], in the production of new energy sources [6], in optics [7], for the purpose of material strengthening [8] and in many other spheres. In the present paper carbon nanotubes are considered from the standpoint of developing advanced carbon membranes for gas separation.

Since the discovery of carbon nanotubes [9] a lot of works have been devoted to the study of both their mechanical properties [10-12] and synthesis [13]. Thus, for example, they are produced not only by means of high-molecular carbon deposition from arc discharge [9], but also in the result of catalytic decomposition of ethanol [14]. In addition, the controlled growth of single-walled carbon nanotubes from the patterned catalysts on substrates is of particular interest [15].

Currently, a special attention is paid to microcirculation of water in nanotube membranes [16], water clusters transport inside a single-walled carbon nanotube [17], water movement through an array of single-walled nanotubes [18], the rheological behaviour of water-based nanofluids in carbon nanotubes [19].

With the application of the Lennard-Jones potential, the interaction between atoms of crystal structures of carbon nanotubes and water molecules is described [20], the effect of the external pressure on a tube's deformation is studied [21] the nature of a hydrogen mixture and carbon monoxide separation is investigated [22].

Using Monte Carlo simulation, $\mathrm{CH}_{4}$ adsorption from mixtures of carbon dioxide and methane in carbon nanotubes [23], as well as the behaviour of $\mathrm{CO}_{2}, \mathrm{~N}_{2}, \mathrm{CH}_{4}$ and $\mathrm{H}_{2}$ molecules [24] were studied and the separation of $\mathrm{H}_{2} \mathrm{~S}-\mathrm{CH}_{4}, \mathrm{CO}_{2}-\mathrm{CH}_{4}, \mathrm{H}_{2} \mathrm{~S}-\mathrm{CO}_{2}$ mixtures and a ternary mixture $\mathrm{CH}_{4}-\mathrm{CO}_{2}-$

\section{${ }^{a}$ Corresponding author: usenko.olesya@yandex.ru}


$\mathrm{H}_{2} \mathrm{~S}$ [25] was simulated. With the help of computer simulation, hydrogen adsorption in carbon nanotubes and slotted pores between them is studied [26].

The research results show that the functionalization of carbon nanotubes not only increases the degree of mixtures separation [27], but also improves their dispersion [28] and increases hygroscopicity [29]. It was also found that mix-matrix membranes (MMMs) with functionalized nanotubes show better performance and a better selectivity from the standpoint of $\mathrm{CO}_{2}$ emission [30].

Carbon nanotubes have opened a new era in the production of multifunctional polymer composites $[13,31-33]$ and are widely used in mix-matrix membranes (MMMs) for separating gas mixtures [3437 , as well as for emitting $\mathrm{CO}_{2}$ from gas mixtures [30,38].

In terms of the model development, the single-walled nanotube is an object which has one of its linear dimensions much smaller than two others, i.e. somewhat of the size (diameter) of the carbon. In other words, the nanotube like fullerene and graphene is the surficial crystal (Figure 1). That is the reason why in a continuous representation of the interaction sources the cumulative effect of the tube is determined by the surface integral.

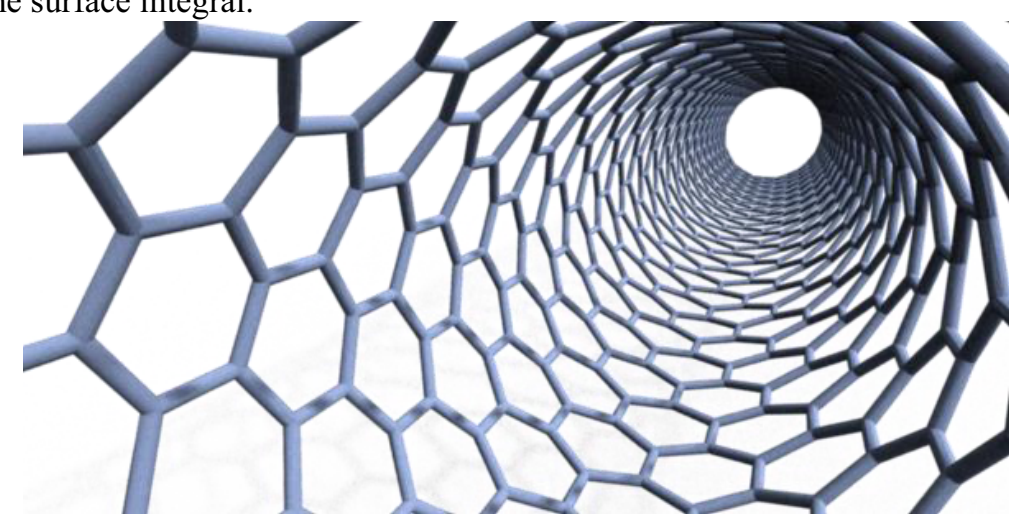

Figure 1. The classic view down the center of a carbon nanotube.

\section{Methods}

The continuum model of the interaction of nanoparticles with molecules was developed in [39-46]. The modification of LJ-potential has the form:

$$
\Phi_{1}(\rho)=4 \varepsilon\left(\frac{\sigma}{\rho}\right) \operatorname{th}\left[\left(\frac{\sigma}{\rho}\right)^{11}-\left(\frac{\sigma}{\rho}\right)^{5}\right]
$$

Where $\rho$ - the interatomic or intermolecular distance; $\sigma, \varepsilon-$ the parameters of the Lennard-Jones potential.

Using the cylindrical coordinates $x=r \cos \varphi, y=r \sin \varphi, z=z$, the squared distance between two points $M$ and $M^{\prime}$. While $M=(x, y, z)$ - the point in the considered volume, $M^{\prime}=\left(x^{\prime}, y^{\prime}, z^{\prime}\right)$ - the point on the surface of the tube:

$$
\rho^{2}=\left(x-x^{\prime}\right)^{2}+\left(y-y^{\prime}\right)^{2}+\left(z-z^{\prime}\right)^{2}=r^{2}+\left(r^{\prime}\right)^{2}-2 r r^{\prime} \cos \left(\varphi-\varphi^{\prime}\right)+\left(z-z^{\prime}\right)^{2} .
$$

The potential (1) will be integrated on the surface of the open nanotubes, i.e. on the lateral surface of a cylinder with a radius equal to the radius of the tube. Then in view of (2) we get 


$$
\int_{S} \Phi_{1}\left(\sqrt{r^{2}+\left(r^{\prime}\right)^{2}-2 r r^{\prime} \cos \left(\varphi-\varphi^{\prime}\right)+\left(z-z^{\prime}\right)^{2}}\right) d s^{\prime} .
$$

The square element $d s^{\prime}=H_{z} d z^{\prime} H_{\varphi} d \varphi^{\prime}$, where $H_{z}=1, H_{\varphi}=r^{\prime}$ - Lame coefficients for the considered cylindrical coordinates.

As the result of integration for the axially symmetric object should not depend on the angle $\varphi$, and the angle in fact is the coordinate of the observation point, then (3) it is rational to have $\varphi=0$.

If (3) is multiplied by the distribution density of the force interaction sources (the distribution density of the carbons on the surface of the tube) $q=\frac{n}{S}$ (where $S$ - total area of the surface of the graphene tube, $n$ - the number of atoms on the surface, $q \approx 28 \mathrm{~nm}^{-2}$ ) and simultaneously have $r^{\prime}=a-$ radius of a nanotube, then we'll derive the expression for the impact potential of all carbon nanotubes:

$$
U(r, z)=a q \int_{0}^{2 \pi} \int_{0}^{\mathrm{h}} \Phi_{1}\left(\sqrt{r^{2}+a^{2}-2 \operatorname{arcos} \varphi^{\prime}+\left(z-z^{\prime}\right)^{2}}\right) d \varphi^{\prime} d z^{\prime} .
$$

Where $h$ - the length of the tube. Moreover, the expression (4) will have the potential (energy) dimension. We will make a note, that if the geometric dimensions of the nanotube $h$ and $a$ are presented in nanometers, then subject to the fact that we have already accepted $[q]=\mathrm{nm}^{-2}$, we will get the potential $U(r, z)$ determined by the dimension parameter $\varepsilon$. The expression (4) can be represented as follows

$$
U(r, z)=4 \varepsilon a q \int_{0}^{2 \pi} \int_{0}^{\mathrm{h}}\left(\frac{\sigma}{\rho}\right) \operatorname{th}\left[\left(\frac{\sigma}{\rho}\right)^{11}-\left(\frac{\sigma}{\rho}\right)^{5}\right] d \varphi^{\prime} d z^{\prime},
$$

where $\rho=\sqrt{r^{2}+a^{2}-2 a r \cos \varphi^{\prime}+\left(z-z^{\prime}\right)^{2}}$.

\section{Results}

With the potential of the nanotube (5), using the methods of classical molecular dynamics it is possible to study the permeability of a single nanotube and the permeability of the materials produced by compacting of the homogeneous or heterogeneous tubes. In addition, it is important to study the issues of the sorption properties of the nanotubes. Some conclusions on these properties can be made for the resultant potential pattern (Figure 2,3).

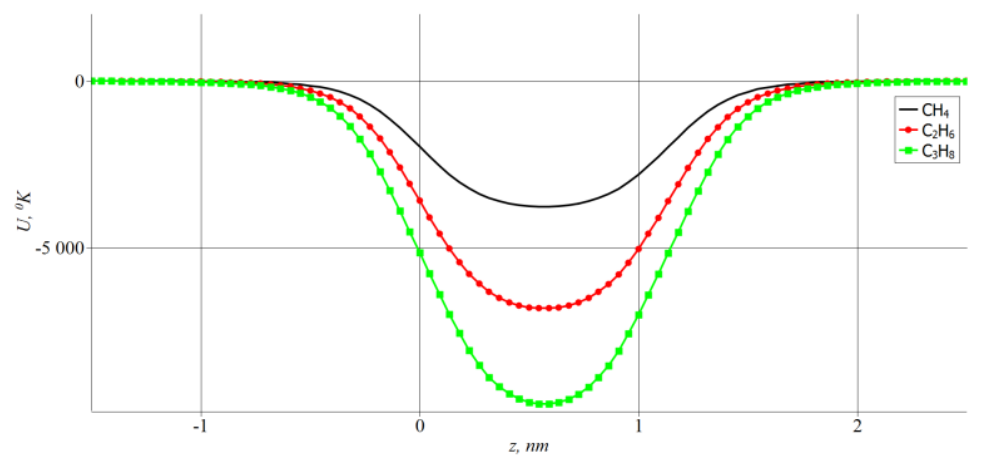

Figure 2. The potential distribution in the linear axial section of the tube. 


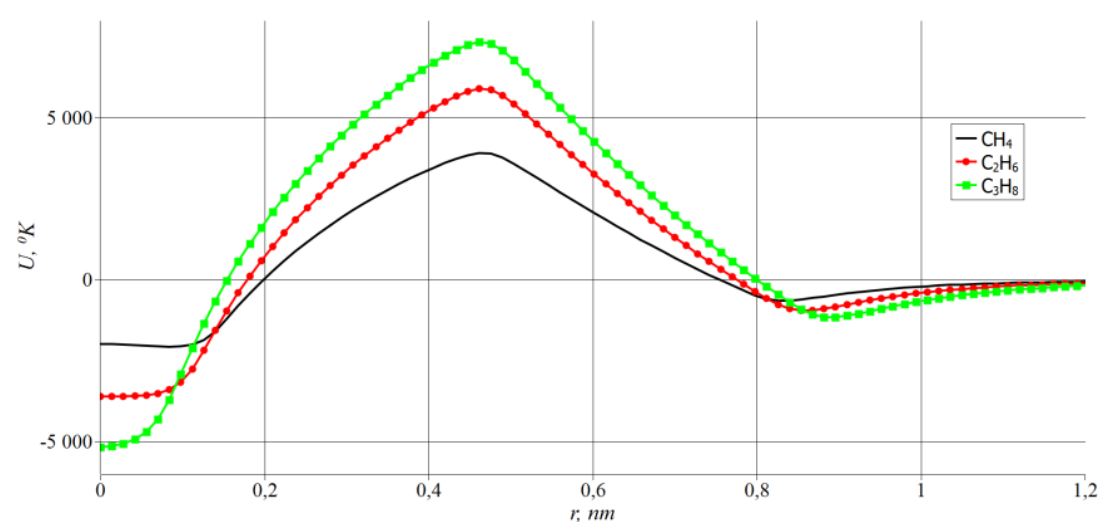

Figure 3. The potential distribution in the central section of the tube that is vertical to its axis.

On Figure $2 z$-coordinate is measured along the axis of the tube. Its entry end is defined by the position $z=0$, exit section $z=1,136 \mathrm{~nm}$. At that the tube radius is $0,475 \mathrm{~nm}$. As can be seen from the graph, the molecule starts sliding down into the potential hole (negative potential values) even before its entry the tube. Out of the tunnel is connected with the rise of the slope of the potential hole and is accompanied by the decreased velocity. Figure 3 shows that the nanoobject under study has two sorption zones located near the axis and outside the cylinder. Moreover, the depth of the central hole is only 1,5 times less than the average of the energy barrier of the graphene layer.

To test the integrated approach based on the continuity distribution of the energy sources on the surface of the tube, the comparison has been done for the values of the velocity of the methane molecule on the tube axis, obtained by the described model, and the data of the common model of the molecular dynamics, wherein the carbons on the surface of the tube have the hexagonal distribution pattern (Figure 1).

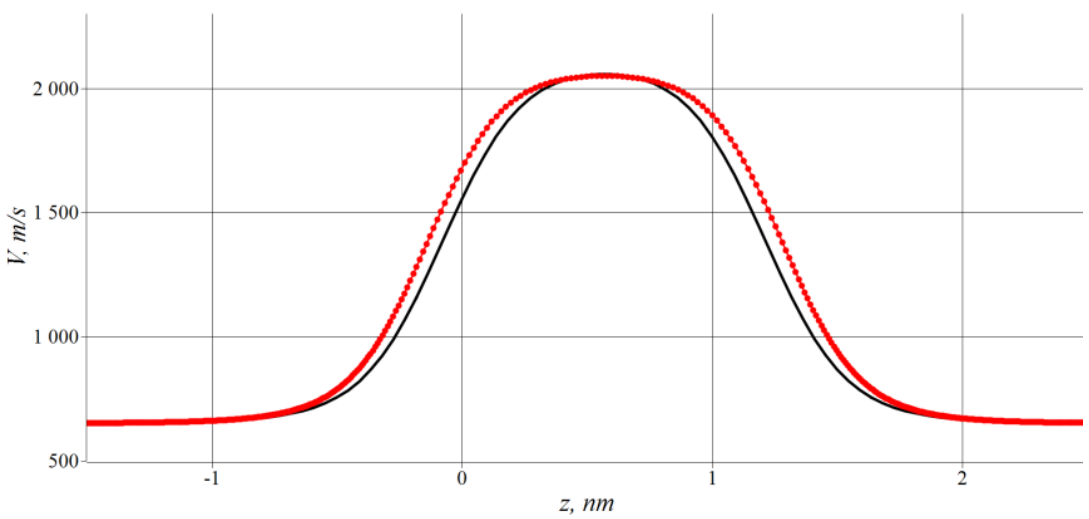

Figure 4. The molecular velocity of methane on the tube axis, "discrete model" - symbols, "continuity model" solid graph.

The lines in Figure 4 can be obtained by integrating the equation of molecular motion, as well as using the energy integral:

$$
m \frac{v^{2}}{2}+U=m \frac{v_{0}^{2}}{2}+U_{0}
$$

Here $V^{2}=u^{2}+v^{2}+w^{2}$ - the square of the absolute speed of the molecule.

As can be seen from Figure 2 and 3, when the molecule starts from a position greater than 3/2 of the tube's radius, the initial value of the potential $U_{0}$ should be taken as zero. 


\section{Movement of the test molecule in the axial plane of the tube}

If the initial velocity vector of the molecule lies in one plane with the axis of the tube, then, due to symmetry, at all subsequent time points the movement will remain flat. In this case, we can write the following equations of motion:

$$
m \frac{d v}{d t}=-\frac{\partial U}{\partial y}, \quad m \frac{d w}{d t}=-\frac{\partial U}{\partial z} .
$$

These equations are to be supplemented by kinematic relations:

$$
v=\frac{d y}{d t}, \quad w=\frac{d z}{d t}
$$

after which the system of four ordinary differential equations can be solved numerically using the Runge-Kutta technology.

If the potential is found (5), the right sides in the equations of motion are known too. These distributions are shown in Figure 5 and 6. The calculated trajectory of the molecule started with a 0.4 $\mathrm{nm}$ offset from the axis of the tube is shown in Figure 7 and its absolute velocity is given in Figure 8 . Comparing Figure 8 with Figure 4, we see that the highest value of velocity is achieved when the molecule moves along the tube axis.

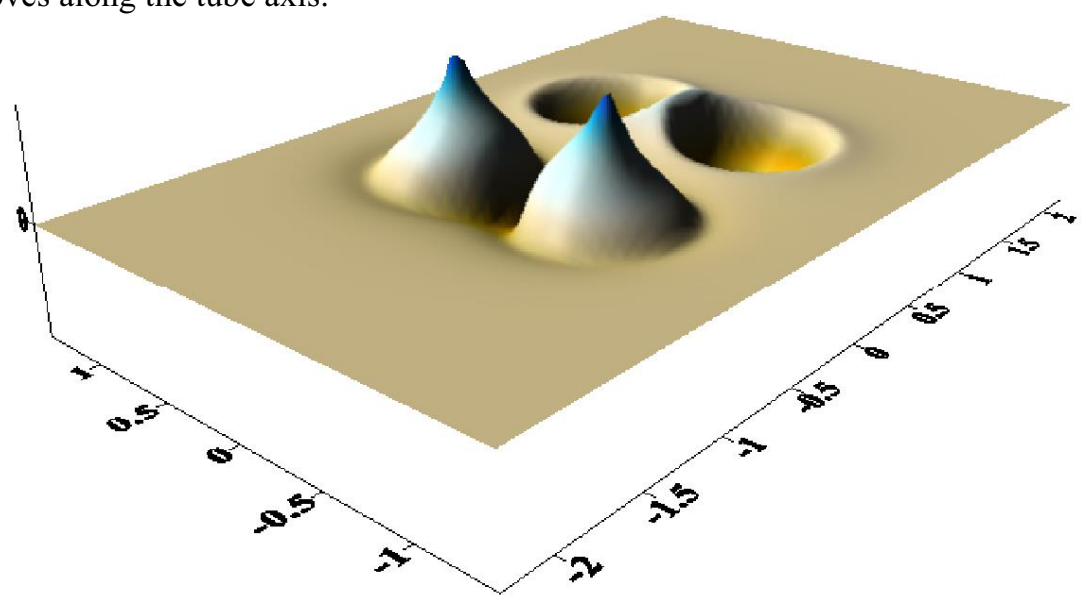

Figure 5. Axial gradient of nanotube's potential.

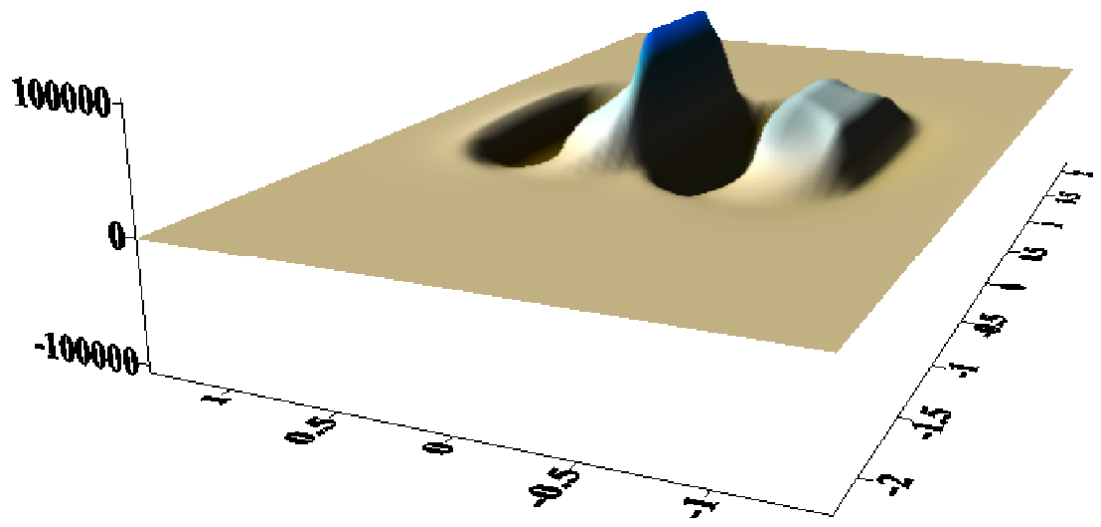

Figure 6. Radial gradient of nanotube's potential. 


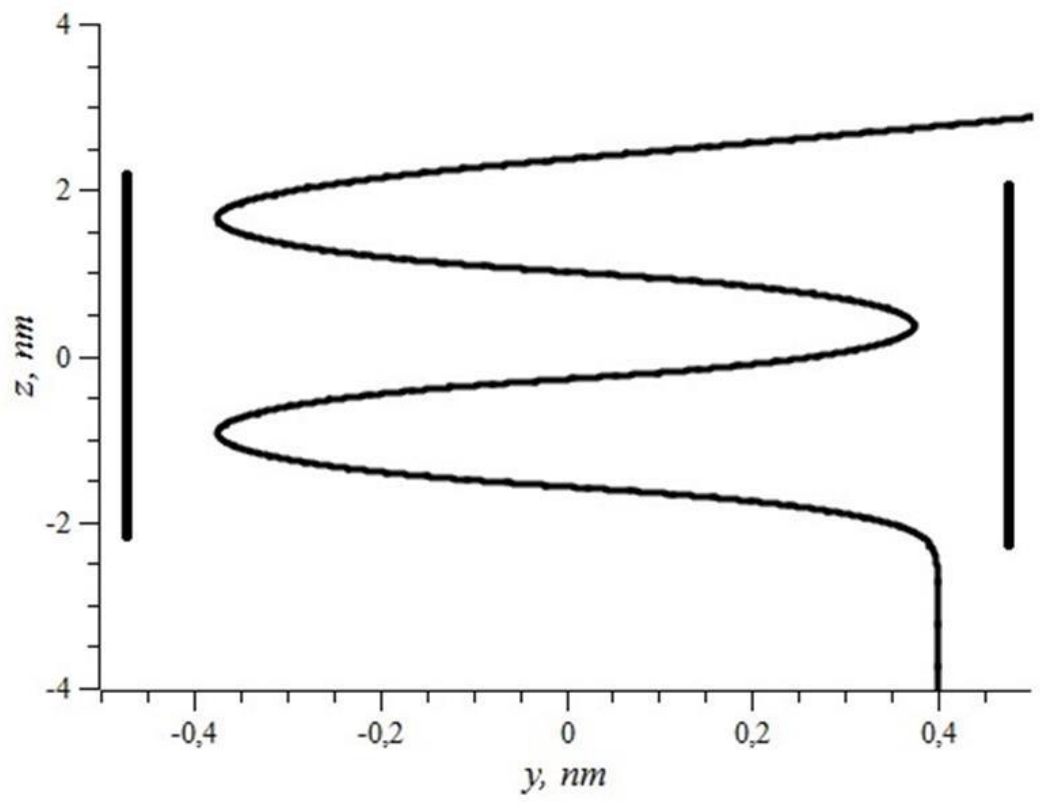

Figure 7. Trajectory of helium molecule started from $\left|y_{0}\right|=0,4 \mathrm{~nm}$ position.

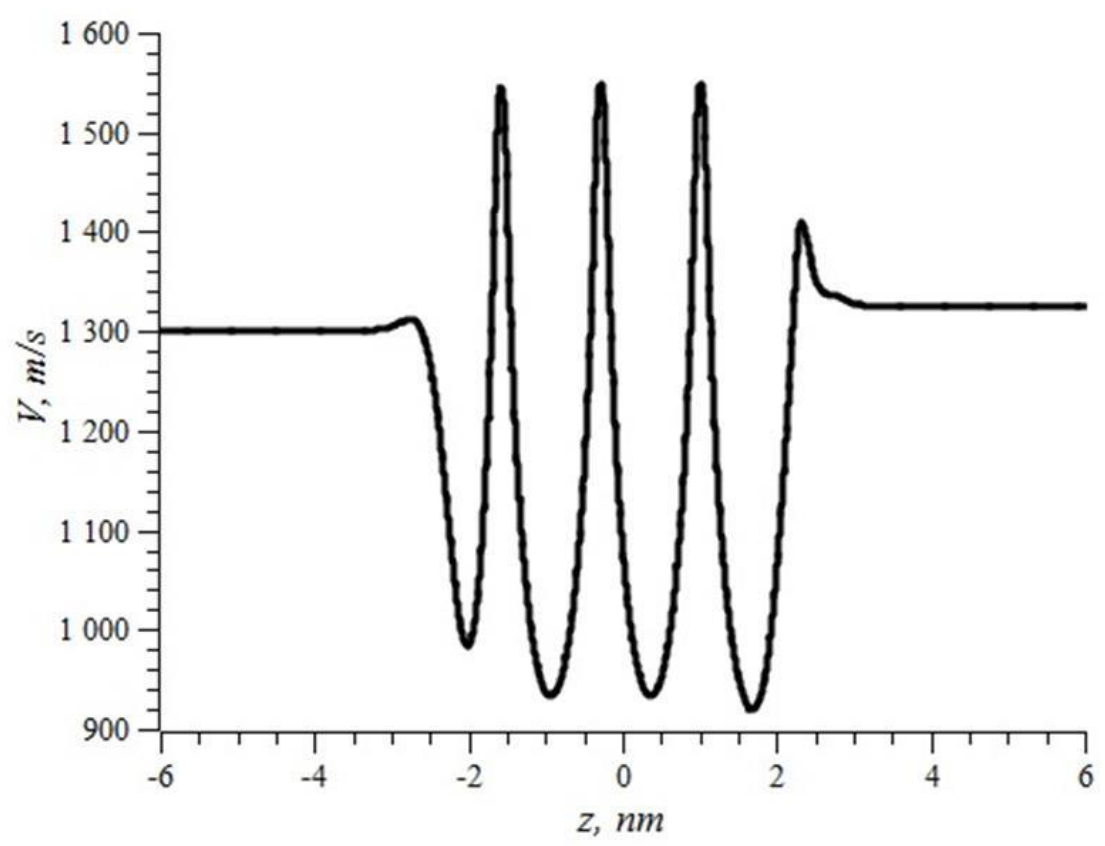

Figure 8. Absolute value of helium molecule velocity depending on axial coordinate.

Spatial motions of molecules result from flat ones if to apply the theorem of conservation of angular momentum for molecular motion in the field of central Van der Waals forces. According to this theorem, the product of the molecule position radius $|y|$ and its peripheral velocity is maintained throughout the motion:

$$
u|y|=u_{0}\left|y_{0}\right|
$$


Thus, if the circumferential velocity at the initial time was not equal to zero $\left(u_{0} \neq 0\right)$, it will be terminal at all subsequent time points.

\section{Discussion}

It is expedient to take the potential of the molecule distant from the nanotube as zero potential energy (energy of interaction). Thus, as seen from the figure the energy of interaction will be negative inside the nanotube. At the same time, no matter what potential value at a distance we take, a molecule that is moving in the direction of the tunnel (nanotube) will be drawn into the tube and activated in its first half-section. After that it will move at a constant velocity (if the tube is long enough). Then there will be the deceleration phase and eventually the molecule will out of the tube with the same velocity as it was at the tube entry. The described scheme of the near-axial motion (the motion without the energy exchange with the atoms of a nanotube) is defined by the part of the LJ-potential, which is responsible for the gravity.

The open nanotube is the tunnel for the molecules to pass through. It is interesting to match the motion pattern inside the other tunnel structures. The wormhole is the tunnel filled with the exotic matter with the negative energy that creates a strong gravitational repulsion preventing the collapse of the hole. The stellar tornado is the tunnel accumulation of the stellar matter with rotation. In this structure, the repulsive forces are the centrifugal forces, and the forces of attraction are the gravitational forces (with a zero integrable potential). In all three cases the energy of interaction within the tunnel is negative and the object that got into the tunnel gains a tremendous velocity.

\section{Conclusions}

Unlike pores in graphene, where the permeability gap is substantially different from the pore size, here a surprising result is observed, which consists in the fact that a nanotube does not actually possess a permeability gap. A molecular object approaching the tube is, in fact, captured by it. The reason for this phenomenon lies in distribution of the interaction potential of the tube interacting with molecules. This potential has a long slope of the potential well in the vicinity of the inlet and outlet sections of the tube.

The solution of the nanomechanics and nanofiltration issues require the development of approaches and methods to determine the integral interaction of nanoobjects of different shapes. The interaction pattern of the molecular structures such as graphite, fullerenes and nanotubes, which are the superficial crystals, is fully defined by integration of the intermolecular interaction potential on the surfaces of these objects. As a result we have the correlation defining the interaction of these molecular structures with the molecules and against each other. As the result, we get relations defining potential interaction of these structures with molecules, after which problems of molecular passage through nanostructures and their sorption can be solved.

\section{Acknowledgment}

This paper was written within the frame of Competitiveness Improvement Program of Tomsk State University and with the financial support of the Russian Foundation for Fundamental Research, grant under Agreement \# 14-01-31365.

\section{References}

1. D. Huang, J. Yu, W. Liu, Z. Li, Z. Yi, J. Wu, Curr. Nanosci., 9, 1 (2013)

2. M. Mohiuddin, D. Arbain, A.K.M. Shafiqul Islam, M. Rahman, M.S. Ahmad, M.N. Ahmad, Curr.

Nanosci., 11 (2015) 
3. H. He, L.A.Pham-Huy, P. Dramou, D. Xiao, P. Zuo, C.Pham-Huy, Biomed. Res. Int., 2013 (2013) 4. W. Hoenlein, F. Kreupl, G. S. Duesberg, A. P. Graham, M. Liebau, R. Seidel, E. Unger, Silicon (2004)

5. W. Hoenlein, F.Kreupl, G.S. Duesberg, A.P. Graham, M. Liebau, R.V. Seidel, E. Unger, IEEE Trans. Compon. Packag. Manuf. Technol., 27, 4 (2004)

6. M.D. Lima, N. Li, M. Jung de Andrade, S. Fang, J. Oh, G.M. Spinks, M.E. Kozlov, C.S. Haines, D. Suh, J. Foroughi, S.J. Kim, Y. Chen, T. Ware, M.K. Shin, L.D. Machado, A.F. Fonseca, J.D. W. Madden, W.E. Voit, D.S. Galvão, R.H. Baughman, Science, 338, 6109 (2012)

7. J. Yu, X. Jiang, D. Zeng, X. Chen, F. Jiao, Z.Peng, Curr. Nanosci., 9 (2013)

8. C. Xiaolan, W. Xiaofei, Curr. Nanosci., 8, 1(2012)

9. S. Iijima, Nature, 354 (1991)

10. E.W. Wong, P.E.Sheehan, C.M. Lieber, Science, 277, 26 (1997)

11. C. Li, T.W. Chou, Int. J. Solids Struct., 40 (2003)

12. S.Her, S.Liu, Curr. Nanosci., 8, 1 (2012)

13. A.L. Martinez-Hernandez, C.Velasco-Santos, V.M. Castano, Curr. Nanosci., 6, 1 (2010)

14. G. Wang, J. Wang, H. Wang, J. Bai, Journal of Environmental Chemical Engineering, 2, 3 (2014)

15. X. Zhou, F. Boey, H. Zhang, Chem. Soc. Rev., 40 (2011)

16. D. Mattia, H. Leese, K. P. Lee, J. Membrane. Sci., 475 (2015)

17. J. Shiomi, S. Maruyama, Nanotechnology, 20, 5 (2009)

18. J.H. Walther, T. Werder, R.L. Jaffe, P. Koumoutsakos, Phys. Rev. E Stat. Nonlin. Soft Matter Phys., 69, 6 (2004)

19. P. Estellé, S. Halelfadl, N. Doner, Curr. Nanosci., 9, 2 (2013)

20. M. Kaukonen, A. Gulans, P. Havu, E. Kauppinen, J. Comput. Chem., 33, 6 (2012)

21. W.B. Lu, B. Liu, J. Wu, J. Xiao, K.C. Hwang, S.Y. Fu, Y. Huang, Appl. Phys. Lett., 94 (2009)

22. C. Gu, G. Gao, Y. Yu, T. Nitta, Fluid Phase Equilib., 194-197 (2002)

23. L. Liu, D. Nicholson, S.K. Bhatia, Chemical Engineering Science, 121 (2015)

24. Q. Sun, M. Wang, Z. Li, Y. Ma, A. Du, Chemical Physics Letters, 575 (2013)

25. F. Gholampour, S. Yeganegi, Chemical Engineering Science, 117 (2014)

26. Q.Y. Wang, J.K. Johnson, J. Chem. Phys., 110 (1999)

27. N. Gilani, J. Towfighi, A. Rashidi, T. Mohammadi, M.R. Omidkhah, A. Sadeghian, Applied Surface Science, 270 (2013)

28. A.L. Ahmad, Z.A. Jawad, S.C. Low, S.H.S. Zein, Curr. Nanosci., 9 (2013)

29. A. Surapathi, H. Chen, E. Marand, J. K. Johnson, Z. Sedlakova, J. Membrane. Sci., 429 (2013)

30. A.L. Ahmad, Z.A. Jawad, S.C. Low, S.H.S. Zein, J. Membrane. Sci., 451 (2014)

31. E.P. Favvas, S.F. Nitodas, A.A. Stefopoulos, S.K. Papageorgiou, K.L. Stefanopoulos, A.Ch. Mitropoulos, Separation and Purification Technology, 122 (2014)

32. M. Nour, K. Berean, S. Balendhran, J.Z. Ou, J.D. Plessis, C. McSweeney, M. Bhaskaran, S. Sriram, K. Kalantar-zadeh, International Journal of Hydrogen Energy, 38, 25 (2013)

33. Z. Zhong, J. Yao, Z. Low, R. Chen, M. He, H. Wang, Carbon, 72 (2014)

34. D. Zhao, J. Ren, H. Li, X. Li, M. Deng, J. Membrane. Sci., 467 (2014)

35. F. Ranjbaran, M. R. Omidkhah, A. E. Amooghin, J. Taiwan. Inst. Chem. E., 49 (2015)

36. M.M. Khan, V. Filiz, G. Bengtson, S. Shishatskiy, Md.M. Rahman, J. Lillepaerg, V. Abetz, J. Membrane. Sci., 436 (2013)

37. R.S. Murali, M. Padaki, T. Matsuura, M.S. Abdullah, A.F. Ismail, Separation and Purification Technology, 132 (2014)

38. Y. Zhao, B.T. Jung, L. Ansaloni, W.S. Winston Ho, J. Membrane. Sci., 459 (2014).

39. A.M. Bubenchikov, M.A. Bubenchikov, A.I. Potekaev, E.E. Libin, Yu.P. Khudobina, Izvestiya vuzov. Physics, 58, 7 (2015)

40. A.I. Potekaev, A.M. Bubenchikov, M.A. Bubenchikov, Russ. Phys. J., 55, 12 (2013)

41. M.A. Bubenchikov, A.I. Potekaev, A.M. Bubenchikov, Russ. Phys. J., 56, 3 (2013)

42. M.A. Bubenchikov, A.I. Potekaev, A.M. Bubenchikov, Russ. Phys. J., 56, 7 (2013) 
43. A.M. Bubenchikov, M.A. Bubenchikov, A.I. Potekaev, A.S. Maslov, V.V. Ovcharenko, O.V. Usenko, Russ. Phys. J., 57, 7 (2014)

44. M.A. Bubenchikov, A.M. Bubenchikov, O.V. Usenko, A.V. Ukolov, IOP CONF. SER. MATER. SCI. ENG, 87, 1 (2015)

45. M.A. Bubenchikov, A.I. Potekaev, A.M. Bubenchikov, Appl. Mech. and Mater., 698 (2015)

46. A.M. Bubenchikov, M.A. Bubenchikov, A.I. Potekaev, E.E. Libin and Y.P. Khudobina, Russ. Phys. J., 57, 8 (2014) 